\title{
Article \\ General Spatial Pattern and Meta-Pattern Model for Problems That Need Analytical Approach in Complex Spatial Systems
}

\author{
Indraja E. Germanaite ${ }^{1}$, Kestutis Zaleckis ${ }^{2}$, Rimantas Butleris ${ }^{1, *}$ and Audrius Lopata ${ }^{1, *}$ \\ 1 Center of Information Systems Design Technologies, Kaunas University of Technology, LT-51368 Kaunas, \\ Lithuania; indraja.germanaite@ktu.lt \\ 2 Faculty of Civil Engineering and Architecture, Kaunas University of Technology, LT-51367 Kaunas, Lithuania; \\ kestutis.zaleckis@ktu.lt \\ * Correspondence: rimantas.butleris@ktu.lt (R.B.); audrius.lopata@ktu.lt (A.L.)
}

check for updates

Citation: Germanaite, I.E.; Zaleckis, K.; Butleris, R.; Lopata, A. General Spatial Pattern and Meta-Pattern Model for Problems That Need Analytical Approach in Complex Spatial Systems. Appl. Sci. 2022, 12, 302. https://doi.org/10.3390/ app12010302

Academic Editors: Yosoon Choi and Antonio López-Quílez

Received: 25 October 2021

Accepted: 24 December 2021

Published: 29 December 2021

Publisher's Note: MDPI stays neutral with regard to jurisdictional claims in published maps and institutional affiliations.

Copyright: () 2021 by the authors. Licensee MDPI, Basel, Switzerland. This article is an open access article distributed under the terms and conditions of the Creative Commons Attribution (CC BY) license (https:/ / creativecommons.org/licenses/by/ $4.0 /)$.

\begin{abstract}
Spatial Pattern (SP) Description, Identification, and Application Methodology (SPDIAM) was developed for describing and detecting spatial economic, social, and environmental phenomena and providing basic information technology (IT) artefacts that can be used for the spatial analysis development using GIS technologies. SPDIAM allows urban planning and design practitioners to describe SP in a computerized manner, identify SP automatically, and apply them in the spatial planning and design domain. In this article, we explain the general SP and spatial meta-pattern model, used in SPDIAM, that is based on the theory of Complex Spatial System (CSS), spatial configuration, and spatial capital concepts and is presented using UML diagrams as standard used for visualization of project models from structure and behavior points of views. The practical experiment of describing and identifying 6 basic spatial meta-pattern values is conducted using the new algorithm that combines Space Syntax method, Visibility Graph Analysis (VGA), and VGA measures to create a computer model of space and to quantify its configuration, which can then be used to handle geographic and geometric data associated with attribute information, to perform spatial, mathematical, and statistical calculations and to visualize SP. The results of the experiment show that the model and the algorithm are appropriate for spatial meta-patterns identification, and the best results can be achieved using VGA measure Isovist Compactness. In the future, general SP and the spatial meta-pattern model can be used to describe and identify complex SP and to solve problems in CSS with the help of the spatial meta-pattern values described in this article.
\end{abstract}

Keywords: pattern analysis; geographic information system; space syntax; network theory; graph

\section{Introduction}

Information systems (IS) research examines more than the technological or social system, or the two side by side; it investigates the phenomenon that emerges when the two interact [1]. IS, as a design science, does not attempt to develop concrete information technologies (IT) applications but, rather, general solution concepts that help to develop the concrete IT applications or specific solution concepts [1].

In our previous article [2], we presented Spatial Pattern (SP) Description, Identification, and Application Methodology (SPDIAM) and SP identification algorithm. SPDIAM allows urban planning and design practitioners to describe SP in a computerized manner, identify SP automatically, and apply them in the spatial planning and design domain. SPDIAM is based on the Space Syntax method and normalized spatial and non-spatial measures and can be used with the statistical social, economic, and environmental indicators, which are related to the urban sustainability and spatial capital. The concepts that were created at the stage of defining SPDIAM can be used for the development of the spatial structure (such as network or grid) analysis tools in Geographic Information System (GIS). Space Syntax approach was chosen as the most appropriate for this research as it: (a) views 
the urban spaces as a complex, constantly interacting network, thus reflecting nature of a city as a complex, dynamic system; (b) uses mathematical methods and mathematical graph model; (c) offers a high variety of theoretically grounded and empirically tested both normalized and not normalized centrality measures, which create a lot of possibilities for spatial configuration analysis and comparison; and (d) could be seen as a tool to evaluate spatial capital [3]. Examples on disciplines where Space Syntax has been applied are: anthropology, archaeology, architecture, urban planning and design, geography, psychology (wayfinding and perception of safety), sociology, criminology, real estate development, and road engineering [4]. Space Syntax was applied in various urban design, strategic planning, and consultancy projects, such as public realm design for Trafalgar Square, the new highway link through the Dutch city of Leiden, the strategic plan for railway stations for North Holland, the use of space syntax in densification strategies in the Norwegian city of Bergen, the master plan of Jeddah, the redesign of Woolwich Squares in London, the regeneration of the areas around King's Cross Station in London, the upgrade of the Old Market Square in Nottingham, the evaluation of the location of the Millennium Bridge in London, and the creation of spatial strategies for the Chinese city of Changchun, among others [5].

In this article, we further analyze the static and dynamic view of SPDIAM [2], e.g., UML class model, that describes SP structure and attributes, and the methods and algorithms to identify spatial meta-patterns in the spatial data sets. The analysis is based on the theory of Complex Spatial System (CSS) as a system of interest of SPDIAM, and CSS represents the spatial entity for which SP is identified. Examples of CSS are regional systems (cities, regions, European Union), urban systems (cities disaggregated to show their structure and working), functional systems (economic (agriculture, resource, manufacturing industry, consumer services, producer services), social, labor market, spatial systems (point patterns (e.g., systems of cities), interactions (commuting flows), networks (traffic, communications), etc. [6]. SPDIAM is based on the spatial configuration and spatial capital [7] concepts, as they closely examine the structure, constitution, and attributes of SP, as well as on detailed research of SPDIAM problems and methods using Design Science Research in IS [1], Complex Spatial System Approach [6], and Algorithmic Approach [8]. As a result, a general SP and meta-pattern model is explained using UML diagrams, and the practical experiment of using this model for the describing and identifying 6 basic spatial meta-pattern values is conducted. For the experiment, the new algorithm using Space Syntax method, Visibility Graph Analysis (VGA), and VGA measures [9] is presented, and the results of the experiment are evaluated. SPDIAM flexibility, expandability, and reusability is demonstrated in comparison with the other spatial analysis methods, such as Cellular Automata, Agent-Based Modeling, Fractal Analysis, or stand-alone Space Syntax.

In the diagrams of this article, the blue color UML classes mean the classes used in SPDIAM, and the white ones are added for the explanatory purpose only, to express the main mechanics, opportunities, and flexibility of the SPDIAM model in general.

\section{Research Background Overview}

\subsection{Complex Spatial System and Algorithmic Approach}

Cities and regions are complex, adaptive, self-organizing systems [8]; thus, urban and regional analysis can be seen as concerned with CSS [6]. CSS defines a spatial entity and is described by many variables, with high levels of interdependence between elements, governed by nonlinear processes and having significant spatial structure [6]. Therefore, the analysis of the separate components does not give the full picture of CSS [10], and the nonlinearities in CSS analysis can arise in a variety of ways: rates of change are non-constant; distance effects involve a power or exponential function (such as in the geographer's gravity model), etc. [6].

Complexity is a general property of CSS that takes the form of hierarchy-a recursive partition of a system into subsystems [1]. Hierarchies have the property of near decomposability: short-term behavior of each subsystem is approximately independent of the 
other components, but the long run behavior of a subsystem depends on other components in an aggregate way, and it simplifies the behavior and the description of CSS [1]. CSS description need not be as complex as the system due to the redundancy property and may contain only a fraction of the connections [6], redundancy results from the fact that there are only a limited number of distinct elementary components, and CSS are obtained by varying their combination [1].

When defining CSS as a system of interest, its elements (people (agents), organizations, and infrastructure) can be assembled into systems in a large number of different ways at different scales [6]. Entities which are components of CSS must be defined and categorized, many of them have to be located in space, and their behavior has to be described over time [6]. Therefore, the important aspects are entitation, number and breadth of categories, and spatial (cartesian coordinates or discrete zone system) and temporal representation of CSS [6].

Urban and regional analysis of CSS can be done through analytical human geography and associated disciplines. An integrated system can be composed of the subsystem models (such as agricultural, industrial, residential location, service delivery, transport, demography, and economic systems [6,8]), and its aims are: (1) handling interdependence of subsystems; and (2) being able to represent the main elements of urban structure. The four stages of CSS analysis [6] are: (1) detailed system of interest and associated analytical problems analysis (this stage is often omitted in modern quantitative geography); (2) statistical analysis; (3) mathematical modeling and formal systems analysis; and (4) application in management or planning.

CSS has to be analyzed in that case when statistical analysis, mathematical modeling, and system analysis methods are not enough; not enough is known about the system to make mathematical analysis possible; the system is too large for feasible analysis; or there are too many variables [6]. In such cases, the computer modeling and computer simulation help, but the CSS modeling task requires a framework for analysis, and SPDIAM [2] offers such a possibility based on the fact that, in CSS, variables are properties of locations which are either averages for the location (e.g., density) or functions of the whole surrounding environment (e.g., accessibilities) [6].

The key to modeling cities as self-organizing systems is to treat them not only as IT artifacts but as processes, which means embedding the model in time [8]. From this perspective, the natural language of modeling is the algorithm (the Algorithmic Approach), since an algorithm is a representation of a process [8]. The original algorithm that captures the generic behavior of a complex self-organizing system was Cellular Automata, conceived in the late 1940s, as simple tool for exploring the nature of dynamical systems [8]. Fractal geometry was invented in 1950 [11]. Agent-based systems appeared near the end of the 1980s, thanks to the combined evolution of artificial intelligence, object oriented programming, and distributed intelligence [12], and other types of algorithms useful for investigating complex adaptive systems were developed, such as classifier systems, artificial neural networks, and random Boolean networks [8]. For the public policy modeling of complexity science, methodologies, such as network science, data mining, and game theory, were applied [13,14]; other urban growth modeling initiatives included linear/logistic regression and decision trees [15].

\subsection{Spatial Pattern and Spatial Captial}

The goal of the Design Science Research in IS is IT artifacts, such as constructs (vocabulary and symbols), models (abstractions and representations), methods (algorithms and practices), instantiations (implemented and prototype systems), and better design theories [1]. In order to fully understand and to create such IT artifacts, we need to analyze the origins of SP emergence and development.

Christopher Alexander noticed [16] that the purpose of the pattern analysis is to detect invariant things in the infinite set of all possible variations [17]. This principle can be applied in any system of interest that is based on the spatial data and needs to be 
analyzed using spatial analysis tools. First, we have to distinguish and name the elements of which the system is made, and then to detect that all parts of the system are made of the different combinations of the same parts, that are members of the same class of the physical structures [16]. Second, we have to name the processes which create and build those patterns in the particular places, and then it is possible to compare them, to make conclusions about what kind of system landscape we have when one or the other process and pattern is used, to detect pluses and minuses, and to select the best one suitable in the particular case [16]. For this purpose, IT artifacts that can describe SP come in handy. Another important insight made by Alexander [16] is that system elements themselves are patterns of relationships, and this morphological law can always be expressed in the same general form $X \rightarrow r(\mathrm{~A}, \mathrm{~B}, \ldots)$ which means that, within a context of type $X$, the parts $\mathrm{A}, \mathrm{B}$, etc., are related by the relationship $r$.

These patterns or relationships or the spatial configuration can play an important role in all three domains of sustainability: environmental, economic, and social [18]. According to the theory of spatial capital [7], urban form generates variations in spatial accessibility and diversity, with effects on social accessibility and diversity, which are possible to measure, whereby, in turn, it is possible to measure variations in urbanity as a sociospatial category. The sustainability compass model [19] uses the similar spatial theory: spatial quality looks at the value relationship between the physical environment and the experiential quality of using it. Thus, spatial configuration (or SP) and spatial quality (or spatial capital) are closely related and must be considered together in any case, given the requirements of CSS.

\subsection{Spatial Pattern Types and Attributes}

Alexander created the pattern language [20], which is composed of the patterns that describe the problem which occurs over and over again in the environment and the core of the solution to that problem. Though Alexander's patterns are primarily dedicated to identify the cities and architecture patterns, they are suitable for the other domains where spatial analysis is required, as well, and they can be used to describe and express fundamental concepts of CSS. Furthermore, Alexander patterns can be described using geographer models [21] and the same concepts and IT artifacts described in this article and in Reference [2]. Most common SP types (overviewed in more detail in Reference [2]) are depicted in Figure 1. In the scope of SPDIAM, the geographer models and Alexander patterns are under consideration, as they have the biggest functional and structural potential in the calculation of the spatial capital.

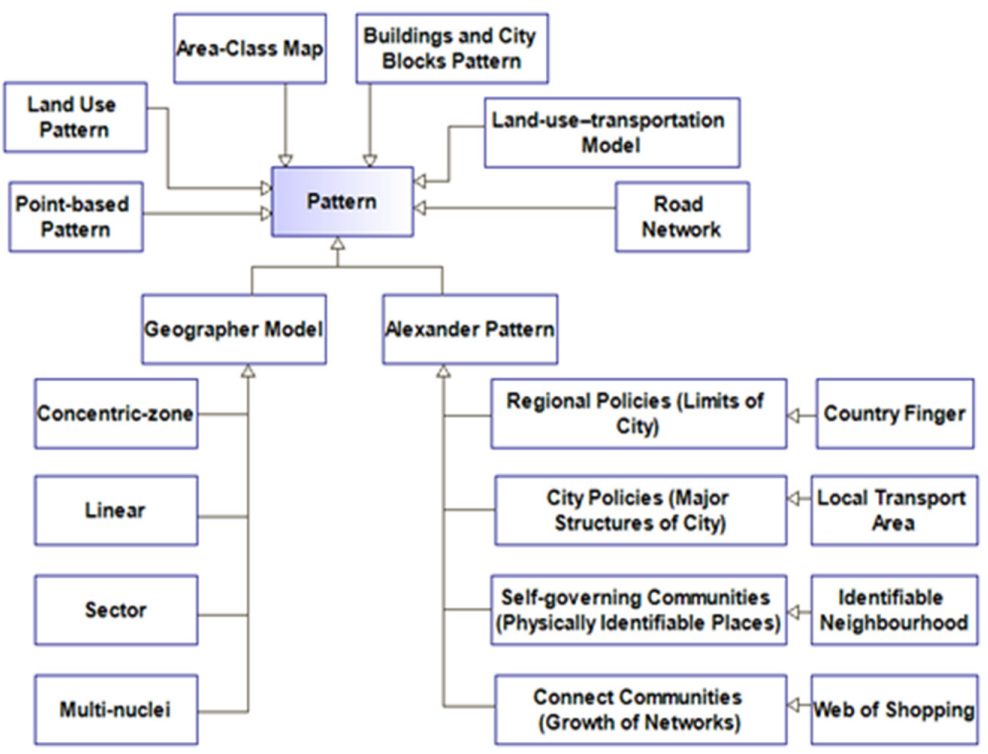

Figure 1. SP types and examples (UML class diagram). 
The term meta-pattern refers to a pattern of patterns [22], and, by analyzing pattern, the interrelated structural, functional, and dynamical aspects of systems, their parts, and their contexts, we draw connections and ultimately make explanatory generalizations [23]. Though the term pattern is usually understandable as spatial or geometric aspects of the system, it can be successfully applied to the relations among parts within a system: description of functional relationships or temporal dynamics of system as a pattern in time [23]. As such, we can expect (1) to develop conceptual constructs arising from the data analyses, which are based on interacting sets of meta-patterns embedded in the system; and, (2) since meta-patterns appear throughout cultural and social contexts, to find transferability of these conceptual constructs across contexts [24]. Thus, as meta-pattern offers many modeling options, SP can be constructed using small indivisible spatial or logical elements (meta-patterns) that later could be used to identify patterns of different scale and complexity [17].

In Ref. [10], characteristics and differences in urban pattern are analyzed to understand the structure of the urban pattern. Spatial parameters that are used to analyze physical space are classified into four categories: basic features of physical structure are geometrical features of the pattern, such as dimensions of the spatial elements; the second category comprises topological characteristics of the physical space; the third category is focused on measures related to the visibility and perception of space; and the last category is complexity of urban pattern that includes the mathematical relationship and hierarchical structure of the spatial systems. As the result, SP identification gives two main insights about spatial structure: (1) values, that give information about spatial characteristics and diversity of urban pattern; and (2) spatial distribution map of changing urban pattern reflects the unique structure of settlements [10].

However, when creating IT artifacts that can be used in several different domains, it is not enough to analyze the characteristics of SP. The main concepts of spatial data, spatial analysis, and spatial entity (or CSS) as a whole should be analyzed, too. Below, there is a short survey of the most characteristic spatial features, with some examples.

In a feature-based GIS, features are the fundamental representation of geographic phenomena, and it is both a real world geographic entity and its digital representation, and shares common attributes, relations, and functions [25]. A feature object may contain six components of a feature-based object [25]: (1) unique identifier; (2) positional or geometric information (depending on the type of the feature and application); (3) non-spatial attribute data (such as names or values of attributes); (4) topological relations among geometric objects (such as boundaries, neighbors, and interior); (5) non-topological relations (nongeometric links between features); and (6) methods embedded in each object.

According to the other classification [26], the main objectives of the spatial information formalisms are to provide specific concepts and notations used in conceptual models to facilitate the following goals: (1) representation of types of basic spatial objects (points, lines, etc.); (2) enumeration of spatial relationships between objects (such as an embankment always being adjacent to a river); (3) description of the evolution of spatial objects over time (birth and death, object shape changes); (4) modeling of multi-representation of objects; (5) description of objects with uncertain boundaries or positions (such as areas of pollution); (6) representation of continuous spatial data (temperature, vegetation cover density); and (7) modeling of the structure of networks (such as water systems).

The features of the spatial data model of geologic map database presented in Reference [27] divide all data into four categories: (1) occurrence (spatial geometry for each geologic feature); (2) descriptor (descriptive information for each feature); (3) concept (essential concepts and definitions essential for querying the database); and (4) symbol (cartographic entities for symbolizing the map on-screen and in print form). The spatial database dedicated to morphological research on architectural heritage [28] divides all data into two categories: (1) spatial data (topographic map, which shows the locations and the shapes of historic architectures, and the relation between them); and (2) non-spatial data (including attribute data and architectural thematic data). These different, but at the same 
time similar, structures for the storage of spatial information must be reflected in the design of the general SP and meta-pattern model.

\subsection{Research Problem and Methods}

There are a number of problems that require additional Design Science Research in IS and make it difficult to develop spatial analysis tools that use SP in CSS analysis. User-defined SP have to conform to CSS problems and are critically dependent upon human cognitive (e.g., creativity) and social (e.g., teamwork) abilities to produce effective solutions [1], so SP must be flexible and configurable. The methods and tools are missing understanding of what can be achieved through the deployment of generic tools and what has to be developed which is specific to that discipline, as many of the spatial problems, across domains, have many common features but are distinguished by the different objectives of the units in those sectors [6]. Because of the high levels of connectivity between different entities and subsystems, there is a strong argument for building a very comprehensive model, but the ideal solution is often impractical, and the partial approaches have to be used [6]. Existing spatial analysis methods and tools can only describe and identify user-defined SP in CSS to a very limited extent, as the constructs created by these methods (such as Space Syntax) are too complex and difficult to understand. Although the taxonomy of SP has been studied extensively, there remains a lack of a clear criterion for SP formalization and automatic identification [29]. Though SP can be regarded as abstract types that allow generalization and a better understanding of the spatial entities, there is no single correct way of classifying SP nor identifying SP types [17,30]. There are already empirically determined urban patterns [20-22,31], but the tool, that would let planning and designing practitioners to identify those SP and have various recommendations and indicators for commercial and social objects placement or utility indicators for public and private sector needs, is still missing. In the existing quantitative GIS tools, the preliminary systems analysis and theorizing cannot be done in activities, such as entitation, scale context, spatial representation, model partial-comprehensive, and appropriate conceptualization [6].

There are two main research methods used in this work: Design Science Research in IS [1] and Complex Spatial System Approach [6]. Both of these methods are defined in Table 1, showing how the process and the goal of the Design Science Research in IS can be aligned with the goal and the process of CSS analysis. The main concept of this work is to achieve (to analyze, design and test in computerized manner) the structural approach as it is defined by CSS theorists and formalize it as the methodology (SPDIAM).

Table 1. Research methods used to define SP problem in CSS.

\begin{tabular}{|c|c|c|}
\hline & Design Science Research in IS [1] & Complex Spatial System Approach [1] \\
\hline Process & $\begin{array}{ll}\text { 1. } & \text { Problem identification and } \\
\text { 2. } & \text { Dotivation } \\
\text { 3. } & \text { Design and development } \\
\text { 4. } & \text { Demonstration } \\
\text { 5. } & \text { Evaluation } \\
\text { 6. } & \text { Communication }\end{array}$ & $\begin{array}{l}\text { 1. Articulation of system of interest } \\
\text { 2. Theory development for system } \\
\text { (needs toolkit of concepts to use } \\
\text { in model-building) } \\
\text { 3. Deployment of appropriate } \\
\text { methods to operationalize the } \\
\text { theory }\end{array}$ \\
\hline Goal & $\begin{array}{l}\text { Artifacts: } \\
\text { - } \quad \text { Constructs } \\
\text { - } \quad \text { Models } \\
\text { - } \quad \text { Instantiations } \\
\text { - } \quad \text { Better design theories }\end{array}$ & $\begin{array}{l}\text { System models: } \\
\text { - } \quad \text { Functional approach } \\
\text { - } \quad \text { Structural approach }\end{array}$ \\
\hline
\end{tabular}

In a general sense, Design Science Research in IS addresses the problems characterized by unstable requirements and constraints, based on ill-defined environmental contexts, complex interactions among subcomponents of the problem, inherent flexibility to change design processes, and design artifacts, and, here, one repeatedly stumbles upon the com- 
plex systems and their behavior [1]. CSS approach could be divided into the functional (static) versus structural (dynamic) approaches [6]. With a functional approach, the forms of organizations and institutions are taken as given, and the emphasis is on the way they function both individually and in a relation to each other, whereas structural approach explores the deeper structures and forces which create these particular forms of organizations [6]. The functional analysis of the city is a statement based on the results of the empirical observation, which has limited explanatory possibilities and no prognostic potential. This is the simplest collection of data on the current situation "who, where, when, how" and so on. A functional approach (for example, a comprehensive plan developed in the territorial planning process) zones territory (CSS) according to its functions, when the structural approach (e.g., SPDIAM) evaluates CSS connections and explores a structure that can perform a variety of functions. More advanced approaches provide a functional and structural analysis of a city modeled as a composition of several networks [32].

SPDIAM with the help of Space Syntax is essentially a structural approach because it evaluates the functional potential of a city through the network configuration (e.g., structure) measures that show the availability, transit of the location, and so on. Space Syntax creates a simulation model based on the structure, connectivity, etc., and it corresponds to the real processes of the city. The functions themselves are shown by statistic data (or indicators), such as building density, points of interest, flows, and so on. Thus, in the first case, we have only the essentially observational data (statistical indicators) without a model, and, in the second case, we have the model and the observational data to check the accuracy and obtain the additional information. For example, a transport model, if presented only as a result of the flows measurement, can be classified as a functional approach, but, if it is based on a model, such as network analysis, it becomes a structural approach. In addition to Space Syntax, there are other methods that belong to the group of structural approaches: Agent-based modeling, Cellular Automata, and Fractal analysis.

The main problem of the existing methods of CSS analysis, and, commonly, even spatial analysis, is that these methods only explore the statistics and functions of CSS, but they lack a structural approach [16] and use quantitative spatial analysis methods instead of qualitative ones. Summarizing all the problems described above, it can be concluded that there is a great need for the methods and tools for a structural approach to CSS and quantitative spatial analysis methods of spatial phenomena (or SP).

\subsection{Research Tools}

In this section, we overview the method and the tools that were used to construct and to test IT artifacts and algorithms for the general SP and meta-pattern model. One of the most important is Space Syntax approach that is used as a computer model of space [33] and address the issues relevant to the formation of the land use strategy and location: to help boost the economy, revitalize central areas, increase social sustainability, and improve cycling and pedestrian access, as it offers an evidence-based approach and a scientific and objective tool for the decision-making and testing strategic interventions and design proposals [34]. Space Syntax is a spatial network analysis method that incorporates the urban morphology and offers the main variables of urban form (accessibility, density, diversity) and the measures of user preference and perception of open space and spatial capital $[2,7,10]$. In Space Syntax, the network made by street lines is modeled as a mathematical graph, and, from this point of view, Space Syntax approach is not unique, as a mathematical graph is used for transport flow modeling by experts of logistics [3]. The essential difference between Space Syntax and other urban modeling approaches appears in a process of graph construction: the logistic modeling chooses crossroads as graph nodes as the main points for logistics in a city [3]. Space syntax marks street axes or segments as nodes, thus focusing on spaces essential for street culture [3].

Space Syntax can operate the axial, convex, or visibility graph analysis maps and provide a range of spatial property parameters derived from the connectivity graph (connectivity, control, integration, and many others) [2]. There are currently three methods 
available for Space Syntax analysis: line-based (axial/segment) analysis, convex-space analysis, and grid-based analysis or VGA [9].

VGA is a means to quantify the configuration of space as regular units which can then be used to identify the relationship of that space to the behavior of the humans that occupy it [9]. For the graph, notation is defined as $G=(V, E)$, where $V$ (the vertices) are the cells of the grid that are part of the graph; $V=\left\{v_{1}, v_{2}, \ldots, v_{n}\right\}$ and $E$ (the edges) are the pairs of mutually visible cells; $E=\left\{e_{1}, e_{2}, \ldots, e_{n}\right\}$. For a specific vertex $v_{i}$, the neighborhood (the other cells that are visible from it) is defined as: $N\left(v_{i}\right)=\left\{v_{j} \mid e_{i j} \in \mathrm{E}\right\}$, where $i$ and $j$ correspond to the indices of the cells in the grid. An isovist is all the points visible from a specific point in space, and, in two dimensions, it can be thought of as a polygon [9]. Apart from providing a way to make the graph, isovists also allow for the creation of different metrics that described the space that is around a cell, such as its area or perimeter [9]. A more detailed overview and an explanation of main Space Syntax concepts and terms can be found in Reference [4].

A range of software [35] has been developed to perform Space Syntax analysis, to handle geographic and geometric data associated with attribute information, to perform spatial, mathematical and statistical calculations, and to visualize the results. In this work, we use the main tool used by the Space Syntax community: DepthmapX [36]. There are 25 VGA metrics that DepthmapX can currently calculate, a mixture of classic graph-theory metrics, metrics borrowed from the urban-scale Space Syntax theories, and some VGAspecific metrics describing local spatial properties [9]. DepthmapX provides a set of global metrics, e.g., for each cell, the values are affected by every other cell in the set, and the aspect that provides this connection in VGA is Visual depth - the least amount of visual steps (a step from a cell to any other immediately visible cell) required to reach another point in the space, and, given that the grid also functions as an undirected graph, the visual metrics can be thought of as topological distance in steps [9].

\section{General Spatial Pattern and Meta-Pattern Model}

\subsection{Essential Assumptions}

Design Science Research in IS is guided by the specific research problem that is usually divided into more manageable subproblems, accepts certain critical assumptions, and requires collection and interpretation of data and creation of IT artifacts [1]. The standard analytical CSS problems, which can be solved by SP, have spatial, functional, and structural background. Where is CSS spatial, economic, social, or environmental capital? What is the pattern of that capital? Is the existing capital optimal? What parts of CSS are more important than the others? Is the planned spatial intervention, such as urban development, in synergy with the existing capital, or will it force to change or even no longer to use the existing capital? There is a relation between such analytical problems, solutions (or SP), and objectives that could be set for CSS. Each solution has to be stated in a such way that it gives the essential field of relationships needed to solve the problem, but in a very general and abstract way, so that the analyst can solve the problem by adapting it to his preferences, as well as the local conditions [20].

In CSS, effectiveness of the developed framework is the extent to which it can lead to efficient modes of analysis that will contribute to problem-solving in these contexts [2]. For it, we have to aggregate the properties, measures, and indicators describing CSS into abstract logical entities, thus creating IT artifacts necessary for solving the tasks of spatial planning and design analysis. Summarizing the assumptions from Section 2 and considerations above, the proposed decomposition of SPDIAM problems is presented in Table 2. It reflects the complexity of the problem applying SPDIAM and can be used as a basis for further development of the proposal. 
Table 2. Composition of SPDIAM problems and solutions.

\section{Question}

1. How to describe CSS? computerized manner?
2. How to describe SP in

\section{Component of Problem}

1.1. How to create CSS model?

1.2. How to describe CSS
analytical problems?

\subsection{How to structure SP properties and values?}

\subsection{How to create user} defined and reusable SP?

2.3. How to make SP configurable and extendable?

\subsection{How to describe CSS} properties?

3. How to identify SP automatically?

\subsection{How to measure CSS properties?}

Solution

Concept (a) partially comprehensive

model

(b) bottom-up model
Spatial Entity (CSS model)
(a) classify and assign solution
(SP) to the problem
Problem
(a) create SP (b) create SP attributes

(c) create SP values

$\begin{array}{ll}\text { (a) create SP based on } & \text { Pattern Type } \\ \text { previously defined } & \text { Pattern Attribute } \\ \text { meta-patterns and SP } & \end{array}$
(a) use SP value measures
(b) create new measures with new measure attributes
Pattern Value, Measure,
Measure Attribute

\section{(a) define quantifiable and} scientifically based CSS measures

(b) perform assessment of CSS based on measures sets
Spatial Entity Attribute,

Measure,

Measure Attribute,

Pattern Value
(a) use whole CSS information
(b) combine different methods
(c) use generic and specific spatial tools
(d) make constructs created by method simple and understandable

\section{Method, Operation Form, Structure}

3.3. How to evaluate identified SP?
(a) use statistical indicators
Indicator
(a) assess CSS at different size and level
(b) compare different CSS
(c) use SP with CSS statistical data

$\begin{array}{ll}\text { 4.1. How to analyze present } & \text { (d) use different dimensions } \\ \text { CSS model? } & \text { (e) associate SP with other } \\ \text { patterns and models } & \text { (f) use quantitative methods } \\ \text { instead of qualitative ones } \\ \text { (g) manage consequences of } \\ \text { interdependence }\end{array}$

4.2. How to indicate directions of CSS future transformation? (a) use CSS measures
Method, Measure, Indicator, Pattern Attribute

\subsection{Spatial Pattern Formalization Model}

The aim of this section is to explain the general model of SPDIAM [2] that is used for SP identification algorithm and let us identify SP in the vector data of the spatial entities. In the scope of this article, the SP model will be used to describe and identify spatial meta-pattern defined in Section 4.

The general assumption of SPDIAM is that there is SP formed by the existing distribution of variables across space [14]. Out of these distributions, certain aggregate quantities can be calculated, such as densities and accessibilities, which form part of the basis for ongoing decisions that will generate changes in CSS, as the whole [6]. SP is a recurring form of a complex physical entity that has spatial structure and can be presented as geometrical 
or topological primitives in a map [2,29]. A structure is a set composed of relations between its elements [12]. SP could be approached on the basis of the theory of urban capitals by Lars Marcus [7] as the four capitals (spatial, economic, ecological, social) are forming the functional background of a city and interacting constantly. The spatial capital is formed by spatial configurations that can significantly affect economic, social, and ecological processes [3]. Therefore, Marcus spatial capital approach could be seen as an expansion and additional explanation to the idea of pattern language by Alexander and makes a background for SP modeling and analysis. Space syntax as a mathematical graph based model is used for SP modeling to catch the spatial capital, but the appropriate selection of the different syntactic measures and indicators could be related to different types of the capitals (economic, social, etc.). The main concepts of the general model to address CSS problems that need analytical approach are presented in Figure 2. There are existing SP in CSS, and these SP can be described by the measures. Every CSS can have different capitals, that can be expressed in terms of spatial configuration, which is the basis for the spatial capital. Spatial configuration can be defined by different spatial characteristics; therefore, spatial configuration consists of SP that consists of different measures.

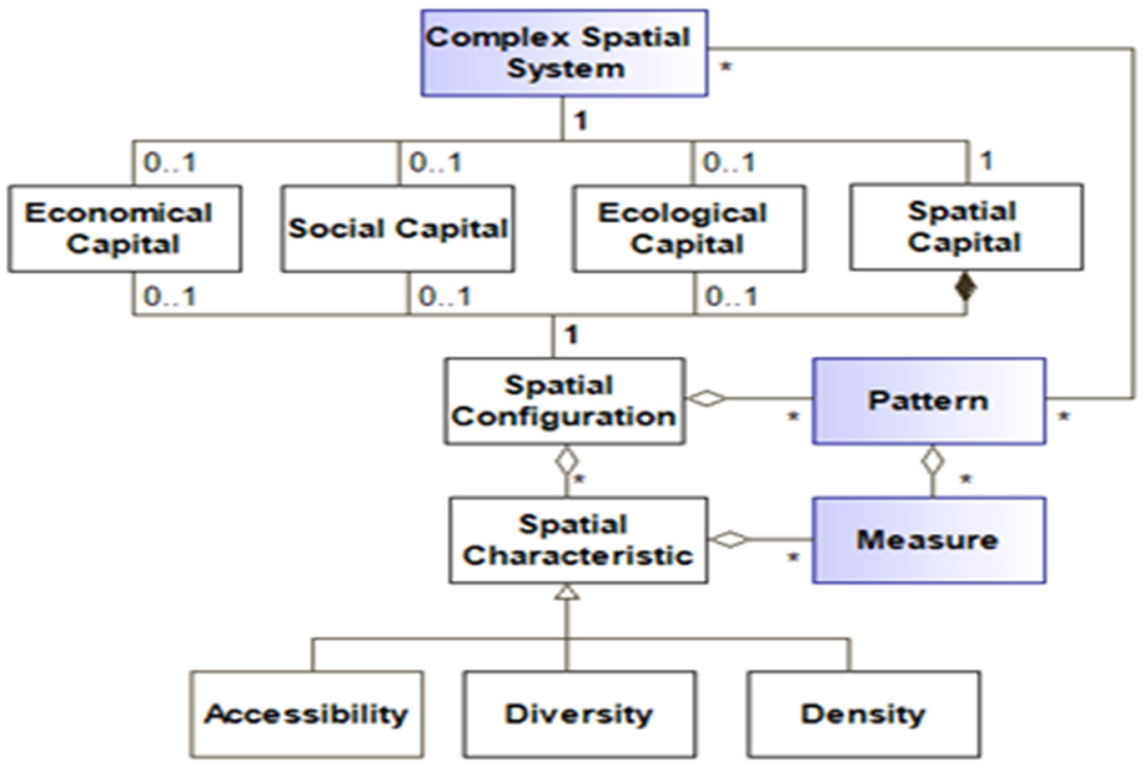

Figure 2. General model to address CSS problems that need analytical approach (UML class diagram).

In UML, cardinality is represented by characters: "..1" (meaning that an instance of the first entity class can be associated with no more than one instance of the second class) or "..*" (meaning that the first entity can be associated with an unlimited number of instances of the second class). A relationship's optionality can be either "0.." (meaning that the relationship is optional) or "1.." (meaning that it is required). Composite aggregation is described as a binary association decorated with a filled black diamond at the aggregate (whole) end. In a composite aggregation, the whole system is responsible for the disposition of its parts, which means that the composite must manage the creation and destruction of its parts. An aggregation is a special type of association in which objects are assembled or configured together to create a more complex object. An aggregation describes a group of objects and how you interact with them. An aggregation association appears as a solid line with an unfilled diamond at the association end, which is connected to the classifier that represents the aggregate [37].

Every SP, as spatial feature, can be defined as consisting of the different kind of attributes (described in Section 2), as presented in Figure 3. 


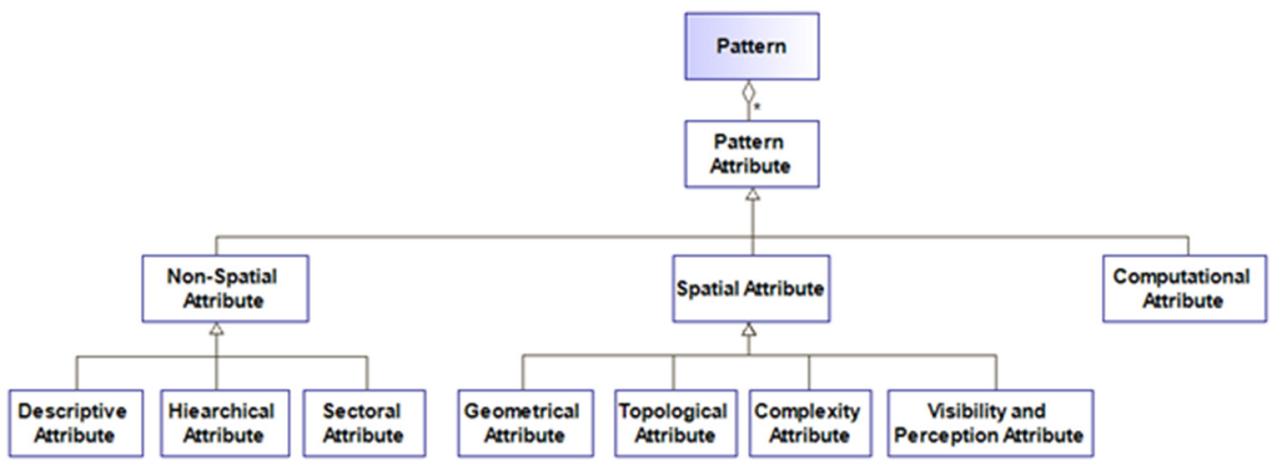

Figure 3. Types of SP attributes (UML class diagram).

The elaborated model of SP is presented in Figure 4, and it has some significant changes. First, the class PAttribute for non-spatial SP attributes is created; it defines descriptive SP attributes (any descriptive SP characteristic, such as origin or scientific justification of SP, etc.), hierarchical SP attributes (such as Configuration), sectoral SP attributes (such as Dimension (spatial, economic, social, or environmental), or SP Context (region, city, district, site). Class Pattern has its own attributes, such as SP Name, Description and Type (SP or meta-pattern). The algorithm for specific SP identification relies on the chosen identification method (such as Space Syntax) (class PatternMethod). The algorithm is described as a sequence of calculated spatial forms that step after step identifies SP. So, for SP spatial attributes, two classes, Form and FAttribute, are created. Class FAttribute defines $\mathrm{SP}$ geometrical attributes (area, perimeter, part size), topological attributes (zone count, part count), visibility and perception SP attributes (visibility, distance), and complexity SP attributes (fractal dimension, lucinarity). Each SP consists of SP values (class PatternValue) that let us identify the specific SP. For example, SP City Layout can have values, such as: co-centric, linear, sector, and so on. Each SP value consists of computational SP attributes (class PatternValueMeasure). Using instances of this class, the real values of the measures that define SP value are kept. The SP identification algorithm compares the return value of $\mathrm{SP}$ identification method with the measures that define SP value.

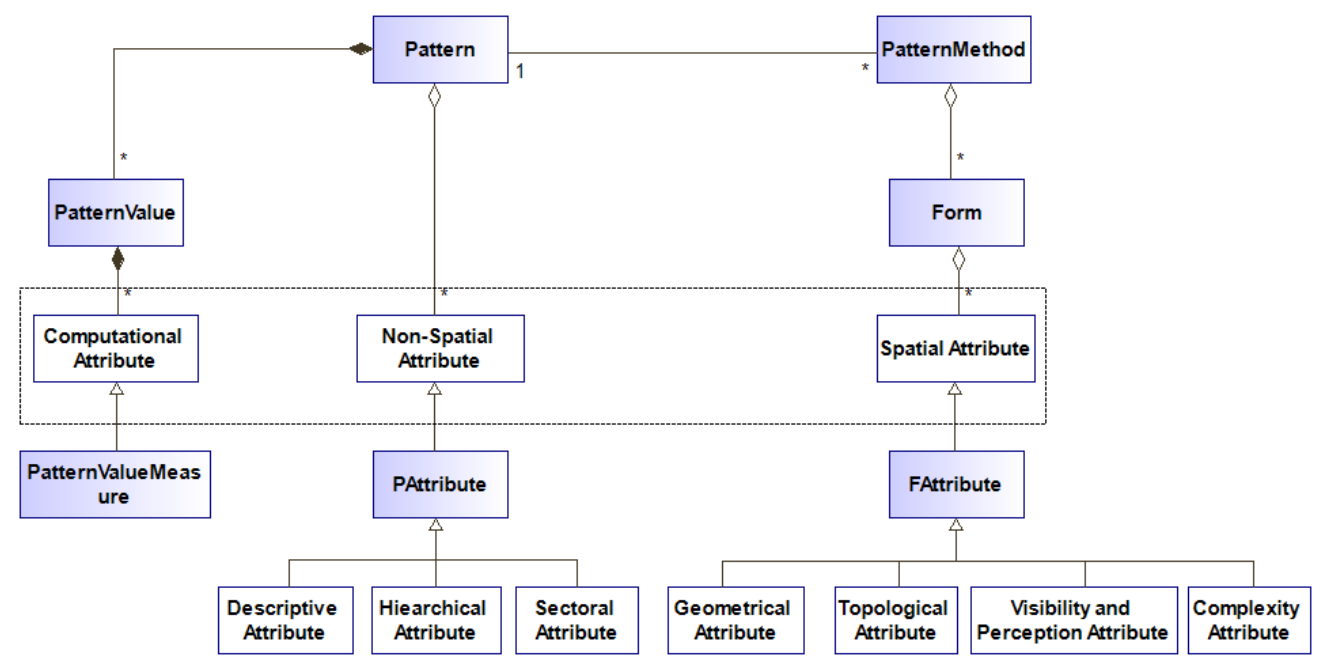

Figure 4. General SP formalization model (UML class diagram).

The detailed idea of SP composition of meta-patterns was presented in Reference [17]. $\mathrm{SP}$ can be constructed of meta-patterns, and the Configuration attribute (instance of class PAttribute) is responsible for this construction. In Figure 5, below, we present 6 spatial meta-pattern values that are used in the spatial meta-pattern description and identification experiment described in Section 4. These basic spatial meta-pattern values depict 6 basic 
geometrical and topological forms that repeat themselves in real-world SP in different proportions.

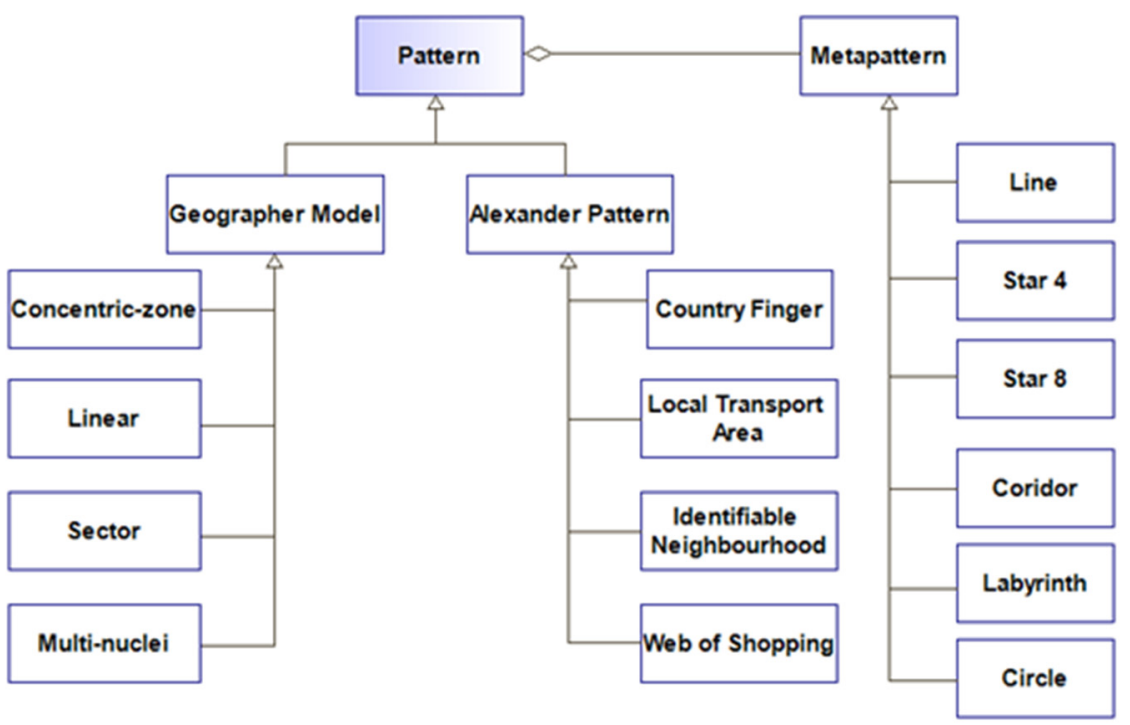

Figure 5. Basic spatial meta-pattern (UML class diagram).

In Figure 6, we present the final SP and meta-patterns model (UML class diagram) which forms the basis of the static description of SPDIAM.

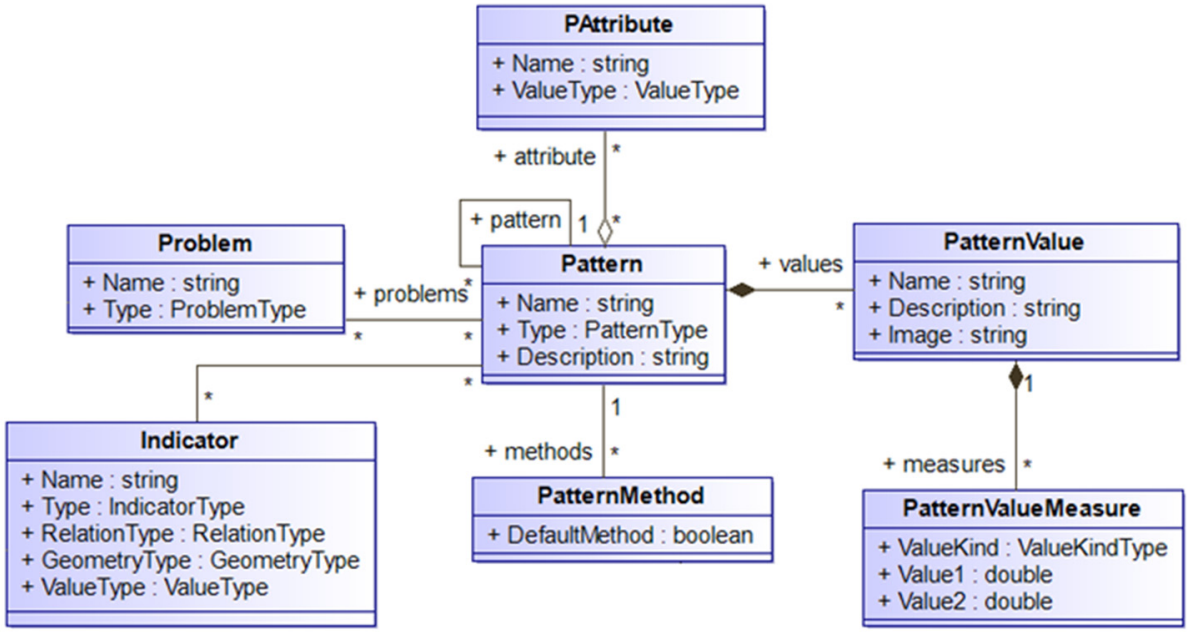

Figure 6. General SP and meta-pattern model (UML class diagram).

\subsection{Spatial Pattern Identification Algorithm}

The new algorithm presented in this article is based on SP identification algorithm used in SPDIAM (phase SP Identification and Evaluation) [2]. First, the area of SP is constructed, using Space Syntax angular segment analysis measures with a certain percentage of maximum values, intersecting them with a grid (size of the cell $200 \times 200$ ); then, SP is formed, identified, and evaluated.

The new algorithm further analyzes the form of SP and uses new Space Syntax structure "Visuality Graph", together with the new VGA measures, to describe and identify the form of SP. The steps of SP identification algorithm (presented in Figure 7) include: (1) selection of SP economical, ecological, or social orientation (PAttribute.Name = 'Dimension'); (2) selection of the proper measures to describe PatternValue (class Measure); (3) tessellation [38] of CSS area to the grid and the assignment of the calculated syntactic values 
of the measures to grid cells (classes Structure and Form); (4) construction of SP area by taking $30 \%$ of the highest value grid cells (classes Structure and Form); (5) identification of SP using VGA and the relation to geographers' and Alexander's pattern typologies (classes PatternValue and PatternValueMeasure); and (6) evaluation of SP using open data indicators (class Indicator). As for spatial meta-pattern, the algorithm is much simpler, and it contains only 2 steps (Figure 8): (1) construction of spatial meta-pattern area; and (2) identification of spatial meta-pattern using VGA measures.

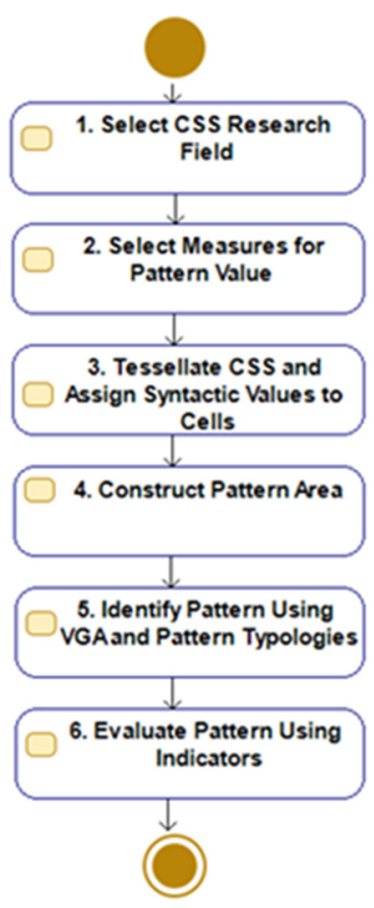

Figure 7. Algorithm of SP identification (simplified).

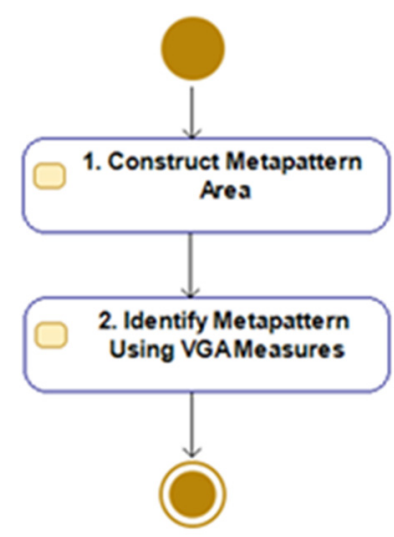

Figure 8. Algorithm of spatial meta-pattern identification (simplified).

\section{Experimental Application of the Model}

The general model of SPDIAM explained in Section 3 was used to conduct the experiment and to describe and identify spatial meta-pattern defined in Section 4. The aim of this spatial meta-pattern identification experiment is to detect the working basic meta-pattern values and measures that can later be used to automatically identify the geographers' and Alexander patterns and to apply them in CSS analysis in other domains. For this purpose, very simple spatial meta-pattern values were chosen to show the clear relationship between their form and numerical values of the selected Space Syntax measures. 


\subsection{Input Data Preparation}

The SPDIAM spatial data preparation routine depends on the method that is selected for SP identification. Space syntax method requires a vector data model, as the spatial network is formed of the roads network layer. The spatial data preparation routine was previously described in Reference [2], but, for spatial meta-pattern identification, this routine is a little different as we do not need data of actual spatial entities (cities or regions). Instead, the 6 spatial entities for 6 meta-pattern values were drawn in Autodesk AutoCAD 2017 and saved as *.DXF files as ideal forms for testing spatial measures. The first spatial entity (Circle) consisted of 778 squares, and, then, the other entities had to fit in the same area of the drawing. The following geometrical structures of spatial entities were prepared: Line, Star4, Star8, Sectoral, Labyrinth, Circle. These primary spatial structures in *.DXF format were transformed to Visuality Graph using DepthmapX (0.5.0.), and each square of the spatial structure became a cell in the Visuality Graph (Figures 9 and 10). The vertices of Visuality Graph were connected to each other if they could be seen from each other without obstruction. As a result, 6 Visuality Graphs were prepared as input data for the experiment.

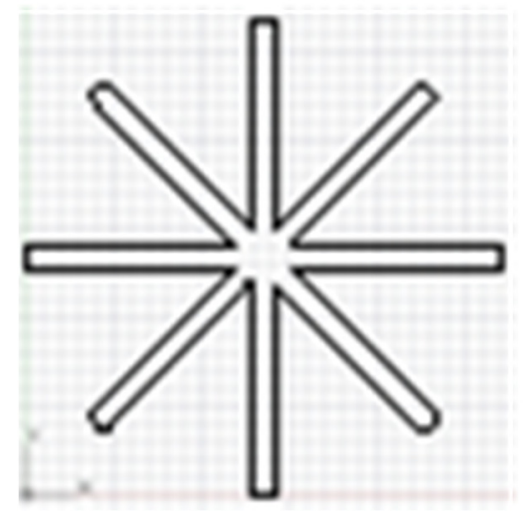

Figure 9. Idealized spatial entity Star8 (AutoCAD drawing, Autodesk AutoCAD).

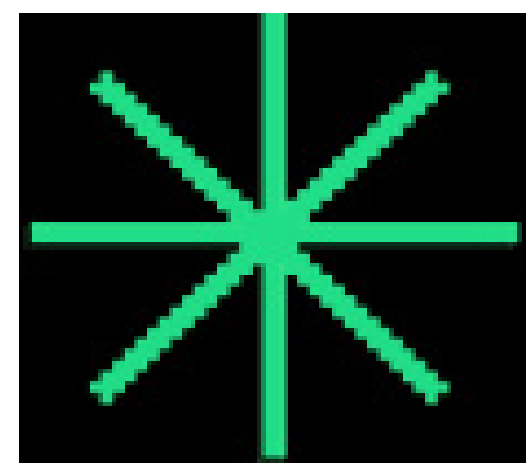

Figure 10. Spatial structure of Star8 (Visuality Graph, DepthmapX).

Such ideal spatial structures present an idealized situation and help to analyze simple geometric forms of SP. Such structures have to be essential (most common) and elementary (the simplest transformation of the selected form). If the need arises, the library of spatial meta-patterns and their values can always be supplemented with the new members; we just have to use the same area of the drawing so that the calculated measure values will be in the same range.

\subsection{Spatial Meta-Pattern Description and Identification}

For the experiment, a basic spatial meta-pattern and its values were described using the instances of UML classes and explanatory attributes of the general SP and meta-pattern model from Section 3, as presented in Table 3. 
Table 3. Description of basic spatial meta-pattern.

\begin{tabular}{|c|c|c|c|}
\hline $\begin{array}{c}\text { Instance_Name: } \\
\text { Pattern }\end{array}$ & Instance_Name: PatternValue & $\begin{array}{c}\text { Type: } \\
\text { PAttribute }\end{array}$ & $\begin{array}{l}\text { Configuration: } \\
\text { PAttribute }\end{array}$ \\
\hline Name & Image & Value & Value \\
\hline \multirow{6}{*}{$\begin{array}{c}\text { 'Basic } \\
\text { Meta-pattern' }\end{array}$} & 'Line' & 'Meta-pattern' & NULL \\
\hline & 'Star4' & 'Meta-pattern' & NULL \\
\hline & 'Star8' & 'Meta-pattern' & NULL \\
\hline & 'Sector' & 'Meta-pattern' & NULL \\
\hline & 'Labirinth' & 'Meta-pattern' & NULL \\
\hline & 'Circle' & 'Meta-pattern' & NULL \\
\hline
\end{tabular}

The structures of basic geometrical meta-pattern values were drawn and analyzed with the aim to select working VGA measures and to test if they can be used for the identification of real SP. VGA measures (listed in Table 4) were selected using these rules: (1) evaluating them by the meaning: what the measure defines or shows on the map; (2) taking those measures that can hypothetically define the shape of the visual field, e.g., the geometrical form of the selected spatial area which are used for SP identification (class Form); (3) testing measures in the VGA spatial meta-pattern description and identification experiment (described in this section); and, finally; (4) evaluating the values of the measures and deciding which measures can be used to define the physical and the functional form of spatial meta-pattern value and which of them can be normalized for later use.

Table 4. VGA measures for basic meta-pattern values identification (measures are taken from Reference [9]).

\begin{tabular}{|c|c|c|c|}
\hline \multicolumn{4}{|c|}{ Instance_Name: Measure } \\
\hline Name & Formula & Component & Description \\
\hline ' $\mathrm{k}^{\prime}$ & 'MAP VARIABLE' & NULL & 'number of cells in system' \\
\hline 'd $\mathrm{d}_{\mathrm{xy}}^{\prime}$ & 'MAP VARIABLE' & NULL & 'distance from cell $\mathrm{x}$ to $\mathrm{y}^{\prime}$ \\
\hline 'v'; 'N' & 'MAP VARIABLE' & NULL & 'cell of the grid'; 'neighborhood' \\
\hline 'e'; ' $\mathrm{E}^{\prime}$ & 'MAP VARIABLE' & NULL & 'pair of mutually visible cells'; 'edge' \\
\hline 'g'; 'c' & 'MAP VARIABLE' & NULL & 'generating point'; 'center of gravity of polygon' \\
\hline 'A'; 'П' & 'MAP VARIABLE' & NULL & 'isovist area'; 'isovist perimeter' \\
\hline 'TD' & ${ }^{\prime} \sum_{y} M I N d_{x y}^{\prime}$ & 'd $\mathrm{d}_{\mathrm{xy}}{ }^{\prime}$ & 'total depth of system' \\
\hline $\begin{array}{l}\text { 'Visual Mean Depth } \\
\text { (VMD)' }\end{array}$ & ' $\frac{T D}{(k-1)} '$ & 'TD, k' & $\begin{array}{l}\text { 'average number of visual steps to reach every other cell } \\
\text { in system' }\end{array}$ \\
\hline ‘RA' & $\frac{2 * V M D}{k-2}$ & 'MD, k' & 'relative asymmetry' \\
\hline ‘ $\mathrm{D}^{\prime}$ & $\frac{\left.2\left(k\left(\log _{2}(k+2) / 3\right)-1\right)\right)}{(k-1)(k-2)}$ & ‘ $\mathrm{k}^{\prime}$ & 'idealized diamond system' \\
\hline ‘RRA' & ' $\frac{R A}{D} \prime$ & 'RA, D’ & real relative asymmetry \\
\hline ‘Point First Moment’ & ${ }^{\prime} \sum_{v_{j} \in N\left(v_{i}\right)} d\left(v_{i}, v_{j}\right) '$ & $` \mathrm{v}, \mathrm{N}, \mathrm{d}$ ’ & 'isovist potentialto spin around' \\
\hline 'Through Vision' & (found in DepthmapX) & 'e, E’ & 'amount of visibility lines that pass through location' \\
\hline
\end{tabular}


Table 4. Cont.

\begin{tabular}{|c|c|c|c|}
\hline \multicolumn{4}{|c|}{ Instance_Name: Measure } \\
\hline Name & Formula & Component & Description \\
\hline 'Isovist Compactness' & $\cdot \frac{4 \pi A}{\Pi^{2}}$ & 'A, $\Pi^{\prime}$ & 'measure of isovist shape that is invariant to its area' \\
\hline 'Drift Magnitude' & $\sqrt{\left(c_{x}-g_{x}\right)^{2}+\left(c_{y}-g_{y}\right)^{2}}$ & 'g, c' & $\begin{array}{l}\text { 'vector from generating point to center of polygon } \\
\text { gravity' }\end{array}$ \\
\hline ‘Isovist Occlusivity' & (found in DepthmapX) & - & 'parts of isovist perimeter' \\
\hline 'Visual Integration' & ' $\frac{1}{R R A_{D}}$ & ‘RRA' & $\begin{array}{c}\text { 'amount transitions needed from graph segment to } \\
\text { reach all segments' }\end{array}$ \\
\hline
\end{tabular}

For the basic meta-pattern identification, Space Syntax method was defined by the class Method, and the VGA measures used were defined by the class Measure, as presented in Table 5. In addition, the instances of the classes Form and Structure with corresponding instances of the class FormMeasure were described. For the structure with the attribute Structure.Type = 'Visuality Graph', the VGA measures from Table 4 were used to identify the values of basic spatial meta-pattern values.

Table 5. Forms and structures for basic meta-pattern.

\begin{tabular}{|c|c|c|c|}
\hline \multicolumn{4}{|c|}{ Instance_Name: Class_Name } \\
\hline $\begin{array}{c}\text { Instance_Name: } \\
\text { Form }\end{array}$ & $\begin{array}{r}\text { Insta } \\
\mathrm{St}\end{array}$ & & $\begin{array}{l}\text { Instance_Name: } \\
\text { Form Measure }\end{array}$ \\
\hline Name & Type & Variant & Description \\
\hline 'Spatial Grid' & ‘Grid' & 'Structure' & NULL \\
\hline ‘Basic Meta-pattern’ & ‘Visuality Graph’ & 'Structure' & $\begin{array}{l}\text { 'Point First Moment', } \\
\text { 'Through Vision',', } \\
\text { Isovist Compactness', } \\
\text { 'Drift Magnitude', } \\
\text { 'Isovist Occlusivity', } \\
\text { 'Visual Integration', } \\
\text { 'Visual Mean Depth' }\end{array}$ \\
\hline
\end{tabular}

Then, the calculation of VGA measures for each basic meta-pattern value was made using DepthMapX, and calculated VGA measures were normalized by dividing their values by the number of the cells of Visuality Graph. The results of the calculation and the visual representation of basic meta-pattern values are presented in Tables 6 and 7.

\subsection{Evaluation of Experiment Results}

In order to evaluate the results of the performed experiment, we have to determine the differences in the numerical scale of the calculated measures values as the form of the spatial meta-pattern changes. The main results of the conducted spatial meta-pattern identification experiment are as follows: (1) Isovist Compactness measure is the most suitable for SP identification as its values show the features of the form of the spatial object and its proximity to the circle using certain clear ranges for different spatial meta-pattern values (measure value approaching to 0 shows Line, and approaching to 1 -Circle metapattern value). MAX and MEAN values of Isovist Compactness can be used potentially as the additional values for more precise SP description; (2) Drift Magnitude measure values also show clear ranges for different spatial meta-pattern values and can be used to identify the degree of the compositional axiality; the bigger value of Drift Magnitude measure is found in more prolonged spatial meta-pattern values; (3) the normalized values of the two measures "Isovist Compactness and Visual Integration" can be used to compare SP of different size and to have the more convenient scale of SP values for SP identification; 
(4) Visual Mean Depth as the measure based on topological distance can be used not normalized and its higher MAX or MEAN values show more Labyrinth, such as spatial meta-pattern structure; and (5) other measures do not demonstrate clear relations between the spatial meta-pattern form and their values, drawing the conclusions from the differences in the numerical scale of the calculated measures.

Table 6. Basic meta-pattern value measures (MAX, MEAN, MIN) and their normalization (N).

\begin{tabular}{|c|c|c|c|c|c|c|c|c|c|c|c|}
\hline Line & Line $\mathbf{N}$ & Star4 & Star4 N & Star8 & Star8 N & Sector & Sector $\mathbf{N}$ & Labyrinth & Labyrinth $\mathrm{N}$ & Circle & Circle N \\
\hline \multicolumn{12}{|c|}{ Point First Moment } \\
\hline 396,615 & 4406.833 & 356,901 & 2176.22561 & 727,968 & 2239.90154 & 658,816 & 1932.012 & 658,816 & 1729.176 & $2,773,020$ & 3564.2931 \\
\hline 270,703 & 3007.811 & 247,634 & 1509.96341 & 278,051 & 855.541538 & 501,121 & 1469.563 & 453,064 & 1189.144 & $2,216,860$ & 2849.4344 \\
\hline 203,295 & 2258.833 & 179,093 & 1092.03049 & 203,804 & 627.089231 & 383,969 & 1126.009 & 5325 & 13.97638 & $1,632,530$ & 2098.3676 \\
\hline \multicolumn{12}{|c|}{ Through Vision } \\
\hline 1980 & 22 & 3452 & 21.0487805 & 6648 & 20.4553846 & 6954 & 20.39296 & 7023 & 18.43307 & 21804 & 28.025707 \\
\hline 1278 & 14.2 & 1154 & 7.03658537 & 970 & 2.98461538 & 2145 & 6.290323 & 1937 & 5.08399 & 6424 & 8.2570694 \\
\hline 0 & 0 & 0 & 0 & 0 & 0 & 0 & 0 & 0 & 0 & 8 & 0.0102828 \\
\hline \multicolumn{12}{|c|}{ Isovist Compactness } \\
\hline 0.126 & 0.126 & 0.136 & 0.136 & 0.134 & 0.134 & 0.505 & 0.505 & 0.543 & 0.543 & 0.997 & 0.997 \\
\hline 0.126 & 0.126 & 0.132 & 0.132 & 0.121 & 0.121 & 0.246 & 0.246 & 0.24 & 0.24 & 0.997 & 0.997 \\
\hline 0.126 & 0.126 & 0.059 & 0.059 & 0.039 & 0.039 & 0.086 & 0.086 & 0.07 & 0.07 & 0.997 & 0.997 \\
\hline \multicolumn{12}{|c|}{ Drift Magnitude } \\
\hline 4409 & 48.98889 & 4196 & 25.5853659 & 4201 & 12.9261538 & 4325 & 12.68328 & 3982 & 10.45144 & 3153 & 4.0526992 \\
\hline 2258 & 25.08889 & 2164 & 13.195122 & 2261 & 6.95692308 & 1792 & 5.255132 & 1637 & 4.186701 & 2098 & 2.6966581 \\
\hline 7 & 0.077778 & 20 & 0.12195122 & 19 & 0.05846154 & 17 & 0.049853 & 22 & 0.056266 & 39 & 0.0501285 \\
\hline \multicolumn{12}{|c|}{ Isovist Occlusivity } \\
\hline 0 & 0 & 8034 & 48.9878049 & 18833 & 57.9476923 & 19733 & 57.86804 & 20675 & 52.87724 & 0 & 0 \\
\hline 0 & 0 & 952 & 5.80487805 & 2923 & 8.99384615 & 7516 & 22.04106 & 8870 & 23.28084 & 0 & 0 \\
\hline 0 & 0 & 0 & 0 & 0 & 0 & 282 & 0.826979 & 400 & 1.049869 & 0 & 0 \\
\hline \multicolumn{12}{|c|}{ Visual Integration } \\
\hline no value & no value & 39.33 & 39.33 & 42.63 & 42.63 & 248.91 & 248.91 & 50.6 & 50.6 & no value & no value \\
\hline no value & no value & 10.36 & 10.36 & 8.6 & 8.6 & 33.61 & 33.61 & 16.54 & 16.54 & no value & no value \\
\hline no value & no value & 9.83 & 9.83 & 7.88 & 7.88 & 8.62 & 8.62 & 3.64 & 3.64 & no value & no value \\
\hline \multicolumn{12}{|c|}{ Visual Mean Depth } \\
\hline 1 & 0.011111 & 1.491 & 0.00909146 & 1.735 & 0.00533846 & 1.619 & 0.004748 & 2.653 & 0.006963 & 1 & 0.0012853 \\
\hline 1 & 0.011111 & 1.461 & 0.00890854 & 1.689 & 0.00519692 & 1.279 & 0.003751 & 1.534 & 0.004026 & 1 & 0.0012853 \\
\hline 1 & 0.011111 & 1 & 0.00609756 & 1 & 0.00307692 & 1 & 0.002933 & 1.102 & 0.002892 & 1 & 0.0012853 \\
\hline
\end{tabular}

Table 7. Basic meta-pattern values (DepthmapX).

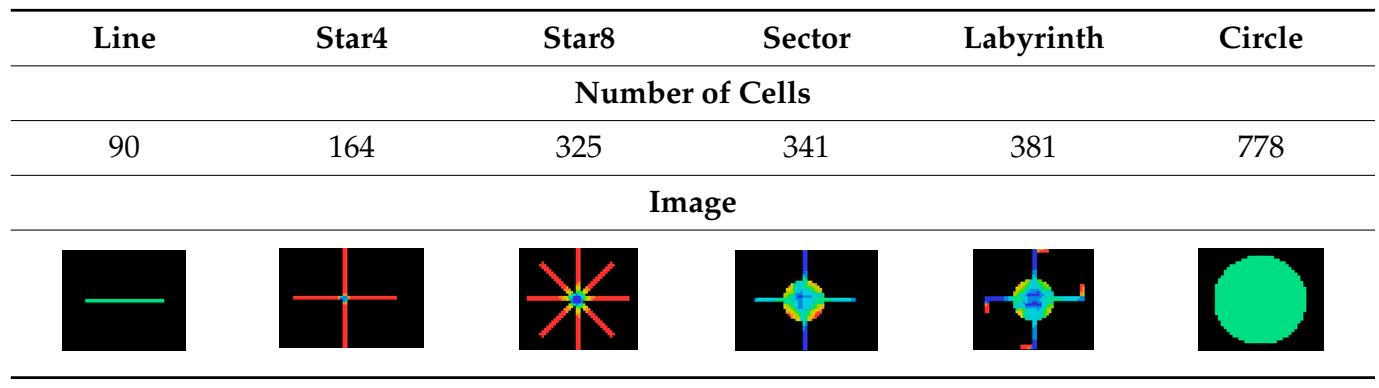

The main conclusion of the experiment is that general SP and meta-patterns model and IT artefacts presented in this article can be used to describe and identify spatial metapatterns in the spatial vector data of CSS, taking into account CSS analysis problems. In the future, more complex SP can be analyzed using the calculated measures and values of simple spatial meta-pattern. For that purpose, city roads (streets) vector GIS data can 
be used to create the partial-comprehensive model of CSS (the detailed procedure of such a model preparation is presented in Reference [2]). Then, the cluster analysis on spatial meta-patterns measures should be performed to create the clusters of measures values combination, in order to use them to identify SP using meta-patterns. Then, VGA should be performed on the parts of CSS to calculate measures and to cluster the parts of the cities into the clusters with assigned meta-pattern values from the previous step. Then, for each CSS part, the percentage dominance of the spatial meta-pattern value should be evaluated, and SP should be assigned using simple rules. The detailed algorithm of this process is the target of the future works. The practical benefits of this research is that the Space Syntax method used in this article to analyze spatial meta-patterns is widely used in various spatial research, modeling, and developing practical processes $[3,5,39,40]$. The presented general spatial meta-pattern and SP model can be used to link Spatial Syntax models to geographer's models, Alexander's models, and so on, thus extending the complexity of this research and providing wider and more diverse usability of CSS analysis. The formalized and computerized description of spatial meta-patterns and SP also allows us to use other methods for the functional analysis of urban structures (or CSS), such as artificial intelligence, neural networks, etc., as these methods also could be based on the analysis of the spatial configurations mentioned in this article.

\subsection{Reusability, Expandability, Flexibility, and Complexity of the General Model}

Whatever technique we choose for modeling complex self-organizing systems, we find ourselves up against a number of methodological problems that not yet been fully resolved [8]. There are four main features of general SP and meta-pattern model that can help to solve CSS problems (described in the Section 3): (1) reusability: SP and metapatterns can be reused for creating new SP, also new spatial meta-patterns can arise based on the existing meta-patterns; (2) expandability: after the practical SP and meta-patterns experiment, knowing the ranges of measure values, we can describe the whole new set of SP and meta-patterns and cover many different geographical models, urban and spatial phenomena and problems using the general SP model and the same principles (UML class model and algorithms); (3) flexibility: existing SP, meta-patterns and algorithms can be improved by using another measures or another SP values, together with the different spatial forms and structures, to refine spatial problems better if the new insights arise while working with SPDIAM; and (4) complexity: SPDIAM algorithm lets us use the different spatial and topological structures (such as Grid, Segment, Convex, and Visuality Graph) for the same SP, using the strengths of each structure. The other spatial analysis methods described in Reference [2], when using them as stand-alone methods, lack these features and need a lot of work and additional IT artefacts to have them.

Cellular Automata, Agent-Based Modeling, Fractal Analysis, and Space Syntax all can be used for creating the simulation models, but the difference is that Cellular Automata, Agent-Based Modeling, and Fractal Analysis show how the process is spreading over time, whereas Space Syntax shows the whole process compressed into one moment of time, as well as the potential of that process; this is the most valuable property of this method. Another important feature is that SPDIAM lets us use different models of spatial entity to follow CSS development over time and to predict changes that can happen in the future (based on Reference [41]). SPDIAM allows the users to create their own SP, their measures, and interpretations of measure values. In addition, SPDIAM can be used to describe other environmental phenomena with a structure, e.g., to identify natural phenomena (natural analysis), to evaluate building interior, etc.

\section{Conclusions}

In this article, the analysis of CSS problems, research methods and properties, together with the spatial data structure, and the deeper analysis of SP and meta-pattern attributes were conducted. As a result, a general model of SP and meta-pattern was explained and presented as an integral part of SPDIAM. Using Design Science Research in IS and Complex 
Spatial System approach (+Algorithmic approach), SPDIAM IT artefacts were detailed concentrating on spatial meta-pattern and VGA analysis. A new VGA-based spatial metapattern identification algorithm was tested with the different VGA measures. The results of the experiment show that the general model and algorithm are appropriate for spatial metapatterns identification, and the best results can be achieved using VGA measure Isovist Compactness. The value ranges of Isovist Compactness measure can be used to identify various SP and spatial meta-patterns in the future. Created IT artefacts can be used for the spatial IS development using GIS technologies and for SP creation and problem-solving in CSS using the structural approach. SPDIAM demonstrates the reusability, expandability, flexibility, and complexity compared to the other stand-alone spatial analysis methods, and, at the moment, there are no known limitations of this model, as it can be easily expanded by creating new spatial meta-patterns and adding new methods, measures, spatial structures, and forms.

In the future, the formalization of the connections between different SP and between SP and meta-patterns, together with the application use cases of identified spatial metapatterns, should be detailed and added to SPDIAM. It would help to clearly define flexible SPDIAM construction possibilities for the creation of an SP library.

Author Contributions: Conceptualization, I.E.G., K.Z. and R.B.; methodology, I.E.G., K.Z., A.L. and R.B.; validation, I.E.G., K.Z., R.B. and A.L.; formal analysis, I.E.G., K.Z. and R.B.; investigation, I.E.G. and K.Z.; resources, I.E.G. and K.Z.; data curation, I.E.G. and K.Z.; writing-original draft preparation, I.E.G., K.Z. and A.L. writing—review and editing, I.E.G. and A.L.; visualization, I.E.G. and K.Z.; supervision R.B. All authors have read and agreed to the published version of the manuscript.

Funding: This research received no external funding.

Institutional Review Board Statement: Not applicable.

Informed Consent Statement: Not applicable.

Data Availability Statement: The data presented in this study are available on request from the corresponding author.

Acknowledgments: We thank the administration of Kaunas university of technology and all colleagues who contributed to the implementation of this study.

Conflicts of Interest: The authors declare no conflict of interest.

\section{References}

1. Chatterjee, S.; Hevner, A.R. Design Research in Information Systems: Theory and Practice; Springer: New York, NY, USA, 2010; pp. 3, $6,11,24,25,49$.

2. Germanaitė, E.; Zaleckis, K.; Butleris, R.; Jarmalavičienè, K. Case Study of Spatial Pattern Description, Identification and Application Methodology. J. Univers. Comput. Sci. 2020, 26, 649-670. [CrossRef]

3. Zaleckis, K.; Tranavičiūte, B.; Grunskis, T.; Gražulevičiūtè-Villeniškè, I.; Vitkuvienė, J.; Sinkienè, J.; Doğan, H.A.; Zaleckis, K. 5 Transformations of the Network on Public Spaces and its Relations to Spatial Social Content. Mod. Public Spaces Lith. Cities 2020, 1, 295-392. [CrossRef]

4. Yamu, C.; van Nes, A.; Garau, C. Bill Hillier's Legacy: Space Syntax-A Synopsis of Basic Concepts, Measures, and Empirical Application. Sustainability 2021, 13, 3394. [CrossRef]

5. van Nes, A.; Yamu, C. Space Syntax Applied in Urban Practice. Introd. Space Syntax. Urban Stud. 2021, 1, 213-237. [CrossRef]

6. Wilson, G. Complex Spatial Systems: The Modelling Foundations of Urban and Regional Analysis; Prentice Hall: Harlow, UK, 2008; pp. 1-4, 6-8, 14,18, 23-25, 96, 97.

7. Marcus, L. Spatial Capital and How to Measure It: An Outline of an Analytical Theory of the Social Performativity of Urban Form. In Proceedings of the Sixth International Space Syntax Symposium, Istanbul, Turkey, 12-15 June 2007. Available online: https://www.researchgate.net/publication/277821851_Spatial_capital_and_how_to_measure_it_An_outline_of_an_ analytical_theory_of_the_social_performativity_of_urban_form (accessed on 15 June 2021).

8. White, R.; Engelen, G.; Uljee, I. Modeling Cities and Regions as Complex Systems. From Theory to Planning Applications; The MIT Press: Cambridge, UK, 2015; pp. 1, 21-23, 32, 129.

9. Koutsolampros, P.; Sailer, K.; Varoudis, T.; Haslem, R. Dissecting Visibility Graph Analysis: The Metrics and Their Role in Understanding Workplace Human Behaviour. In Proceedings of the 12th Space Syntax Symposium, Beijing, China, 8-13 July 2019.

10. Bölen, F.; Kaya, H. Urban DNA: Morphogenetic analysis of urban pattern. Int. J. Archit. Plan. 2017, 5, 10-41. [CrossRef] 
11. Batty, M.; Longley, M. Fractal Cities-A Geometry of Form and Function; Academic Press: London, UK, $1994 ;$ p. 5.

12. Langlois, P. Simulation of Complex Systems in GIS; John Wiley \& Sons Inc.: Hoboken, NJ, USA, 2011; pp. 1-6.

13. Furtado, B.; Fuentes, M.; Tessone, C. Policy Modeling and Applications: State-of-the-Art and Perspectives. Complexity 2019, 2019, 5041681. [CrossRef]

14. Samson, G.L.; Joun, L.; Ajibola, A.S. Mining Complex Spatial Patterns: Issues and Techniques. J. Inf. Knowl. Manag. 2014, 13, 4-7. [CrossRef]

15. Musa, S.I.; Hashim, M.; Reba, M.N. A review of geospatial-based urban growth models and modelling initiatives. Geocarto Int 2016, 32, 813-833. [CrossRef]

16. Alexander, C. The Timeless Way of Building; Oxford University Press: New York, NY, USA, 1979; pp. 82-93.

17. Germanaite, E.; Butleris, R.; Zaleckis, K. How to Describe Basic Urban Pattern in Geographic Information Systems. Commun. Comput. Inf. Sci. 2018, 920, 153-163. [CrossRef]

18. Hillier, B. Spatial Sustainability in Cities: Organic Patterns and Sustainable Forms. In Proceedings of the 7th International Space Syntax Symposium, Stockholm, Sweden, 8-11 June 2009.

19. BUUR. The Sustainability Compass-A Tool for Analysing and Orienting Urban Scale Projects. 2014. Available online: http: / / sustainabilitycompass.eu/theory / (accessed on 11 June 2021).

20. Alexander, C. A Pattern Language; Oxford University Press: New York, NY, USA, 1977; pp. 10-13.

21. Major, M.D. The Syntax of City Space: American Urban Grids; Routledge Books: New York, NY, USA, 2018.

22. Volk, T. Meta-Patterns; Columbia University Press: New York, NY, USA, 1995; pp. 1, 51, 99.

23. Volk, T.; Bloom, J.W. The Use of Meta-patterns for Research into Complex Systems of Teaching, Learning, and Schooling. Part I: Meta-patterns in Nature and Culture. Complicity Int. J. Complex. Educ. 2007, 4, 25-43. [CrossRef]

24. Bloom, J.W.; Volk, T. The Use of Meta-patterns for Research into Complex Systems of Teaching, Learning, and Schooling. Part II: Applications. Complicity Int. J. Complex. Educ. 2007, 4, 45-68. [CrossRef]

25. Tang, Y.; Adams, T.M.; Usery, E.L. A Spatial Data Model Design for Feature-Based Geographical Information Systems. Int. J. Geogr. Inf. Syst. 1996, 10, 643-659. [CrossRef]

26. Pinet, F. Entity-relationship and object-oriented formalisms for modeling spatial environmental data. Environ. Model. Softw. 2012, 33, 80-91. [CrossRef]

27. Soller, D.; Berg, T. The U.S. National Geologic Map Database Project: Overview E Progress; Springer: Cham, Switzerland, 2006. [CrossRef]

28. Xiaoqian, W.; Weimin, G.; Jia, L. Application of spatial database technology of GIS to morphological research on architectural heritage. In Proceedings of the 2010 International Conference on Computer Application and System Modeling (ICCASM 2010), Taiyuan, China, 22-24 October 2010; pp. 502-506. [CrossRef]

29. Yan, X.; Ai, T.; Yang, M.; Yin, H. A graph convolutional neural network for classification of building patterns using spatial vector data. J. Photogramm. Remote Sens. 2019, 150, 259-273. [CrossRef]

30. Marshall, S.; Gong, Y. WP4 Deliverable Report: Urban Pattern Specification; Bartlett School of Planning, University College: London, UK, 2009; Available online: http:/ /www.suburbansolutions.ac.uk/documents/WP4DeliverableReportNov2009.pdf (accessed on 14 July 2021).

31. Borgatti, S.P.; Elerett, M.G. Models of core/periphery structures. Soc. Netw. 2000, 21, 375-395. [CrossRef]

32. Jguirim, I.; Brosset, D.; Claramunt, C. Functional and Structural Analysis of an Urban Space Extended from Space Syntax. In Proceedings of the GIScience 2014, Vienna, Austria, 24-26 September 2014. Available online: https:/ /www.researchgate.net/publication/265521103_ Functional_and_Structural_Analysis_of_an_Urban_Space_Extended_from_Space_Syntax (accessed on 12 June 2021).

33. Jiang, B.; Claramunt, C. A Comparison Study on Space Syntax as a Computer Model of Space. August 1999. Available online: https://www.researchgate.net/publication/2466998_A_Comparison_Study_on_Space_Syntax_as_a_Computer_Model_ of_Space (accessed on 24 August 2021).

34. Charalambous, N.; Mavridou, M. Space Syntax: Spatial Integration Accessibility and Angular Segment Analysis by Metric Distance (ASAMeD). Access. Instrum. Plan. Pract. 2012, 1, 57-62.

35. Gil, J.; Varoudis, T.; Karimi, K.; Penn, A. The Space Syntax Toolkit: Integrating depthmapX and Exploratory Spatial Analysis Workflows in QGIS. In Proceedings of the 10th International Space Syntax Symposium, London, UK, $13-17$ July 2015.

36. Varoudis, T. Multi-Platform Spatial Network Analysis Software. 2014. Available online: https://varoudis.github.io/depthmapX/ (accessed on 3 May 2021).

37. UML as a Data Modeling Notation, Part 1. Available online: https://tdan.com/uml-as-a-data-modeling-notation-part-1/8457 (accessed on 21 June 2021).

38. Huisman, O.; de By, R. Principles of Geographic Information Systems: An Introductory Textbook; ITC: Enschede, The Netherlands, 2009; pp. 85-87.

39. Laboratory, S.S. Research Projects; Bartlett School of Planning, University College: London, UK, 2021. Available online: https: / / www.ucl.ac.uk/bartlett/architecture/research/space-syntax-laboratory/research-projects (accessed on 12 May 2021).

40. Limited, S.S. Our Projects \& Clients. Space Syntax Limited. 2021. Available online: https://spacesyntax.com/cities-regions/ cities-regions-landing-page/ (accessed on 10 April 2021).

41. Al Sayed, K.; Turner, A.; Hanna, S. Cities as Emergent Models: The Morphological Logic of Manhattan and Barcelona. In Proceedings of the 7th International Space Syntax Symposium, Stockholm, Sweden, 8-11 June 2009. 